\title{
High-dose-rate interstitial brachytherapy in recurrent head and neck cancer: an effective salvage option
}

\author{
Rajendra Bhalavat, MD!, Vibhay Pareek, DNB!, Manish Chandra, DNB!', Lalitha Nellore, DNB!', Karishma George, DNB!, \\ Dipalee Borade, DNB', Ketan Kalariya, DNB!', Zaiba Moosa, DNB', Amrita Srivastava, DNB', Navaneeth Reddy, DNB', \\ Ankita Kapoor, DNB', Darshana Kawale, DNB', Nandakumar P, MSc², Pratibha Bauskar, MSc ${ }^{2}$ \\ IRadiation Oncology Department, Jupiter Hospital, Mumbai, Maharashtra, ${ }^{2}$ Radiation Physics Department, Jupiter Hospital, Mumbai, \\ Maharashtra, India
}

\begin{abstract}
Purpose: High-dose-rate (HDR) interstitial brachytherapy has an established role in head and neck malignancies and offers good survival rates; however, there is scant data on improved local control (LC) and treatment-related complications in recurrent cases. We present our results in patients with recurrent head and neck cancers treated with HDR interstitial brachytherapy.

Material and methods: Twenty-five patients with recurrent head and neck cancers were treated with HDR interstitial brachytherapy using Iridium 192 between 2009 and 2016. Of these, 75\% received radical brachytherapy, and $25 \%$ received external beam radiation therapy (EBRT) followed by brachytherapy boost. Treatment sites included oral cavity $(15 / 25)$ and oropharynx (10/25). Median dose of 4.5 Gy was administered twice per day, with median total brachytherapy dose of $40.5 \mathrm{~Gy}$ in radical and 27 Gy for EBRT cases.

Results: With median follow-up of 25 months, 4 local recurrences were observed within first year of follow-up. Two-year local control and overall survival outcomes for the entire group were $75 \%$ and $68 \%$, respectively. Local control rate with radical BRT vs. BRT as a boost following EBRT was found to be significant (2-year LCR 62\% vs. 85\%; $p<0.02$ ). Dosimetric assessment revealed $\mathrm{D}_{90}-4.08 \mathrm{~Gy}, \mathrm{~V}_{100}-94.1 \%, \mathrm{~V}_{150}-24.7 \%$, and $\mathrm{V}_{200}-10.1 \%$. Xerostomia, altered taste, and dysphagia were the major complications commonly grade 1 and 2 . Grade 3 toxicity was only $2 \%$. Pre-treatment volume $>85 \mathrm{cc}$ had a negative impact on overall survival ( 26 months vs. 12 months; $p=0.02)$, and interval time between primary and recurrence more than 15 months had an impact on the local control rate $(p<0.01)$.

Conclusions: Results of HDR interstitial brachytherapy have shown acceptable local control and overall survival rates along with tolerable toxicities and morbidity in recurrent head and neck cancers.

J Contemp Brachytherapy 2018; 10, 5: 425-430 DOI: https://doi.org/10.5114/jcb.2018.78995
\end{abstract}

Key words: HDR brachytherapy, head and neck cancer, recurrent tumor.

\section{Purpose}

Head and neck malignancy management has seen many advancements; nevertheless, approximately $20-50 \%$ of patients are diagnosed with loco-regional recurrence within first two years [1,2]. Surgery forms the major treatment modality but can be possible in only $20 \%$ patients, leading to overall 5-year survival of $20 \%$ to $30 \%$ $[3,4,5,6,7]$. External beam radiation therapy (EBRT) can lead to severe local toxicities in view of reirradiation to the primary. High-dose-rate brachytherapy (HDR-BRT) however, can deliver a high dose directly to the target volume, and provides the advantage of rapid dose falloff, thereby allowing for sparing of normal tissue $[8,9,10]$.
With the advent of 3DCRT (3D conformal radiation therapy) and IMRT (intensity modulated radiation therapy), as more conformal treatment techniques, there has been a rise in the publications presenting results with the use of these treatment techniques, demonstrating the survival outcomes and toxicity profiles associated with reirradiation in head and neck malignancies. However, in most cases, even these techniques cannot prevent the dose fall off to the surrounding normal tissue, thus leading to acute and late toxicities, and sometimes even compromising the doses to target volumes itself leading to future recurrences and failures.

High-dose-rate brachytherapy offers significant advantages over conventional LDR (low-dose-rate) brachy- 
therapy by reducing concerns of radiation safety for the staff during hospitalization and provides the clinician with a greater control over the dose distribution by using computer optimized dwell times within the individual catheters. With HDR-BRT, dosimetrically equivalent or superior outcomes compared to LDR brachytherapy and EBRT for gynecological tumors and other locations have been achieved [11,12]. However, the same data in head and neck malignancies and the use of interstitial brachytherapy is minimal. In this study, we present our results and experience with retrospective analysis demonstrating our recent experience in the use of HDR-BRT in management of recurrent head and neck malignancies.

\section{Material and methods}

\section{Patient characteristics}

From January 2009 to June 2016, twenty-five patients with histopathologically proven recurrent head and neck malignancies were treated with HDR-BRT at Jupiter Hospital, Thane, India. The primary treatment characteristics and patient characteristics are shown in Table 1. Most of the patients had recurrence in the region of previous EBRT or surgery. Out of these 25 patients, 10 were recurrences in oral cavity and 15 in the oropharynx. The patients were evaluated for surgery and found to be ineligible for varied reasons, therefore chosen for radiation therapy with HDR-BRT. There were 14 males and 11 females who underwent reirradiation with HDR-BRT. The median age of these patients was 57 years (range: 34-76 years). In view of larger primary tumors, the patients underwent external beam radiation first, followed

Table 1. Primary treatment characteristics

\begin{tabular}{lc} 
Parameters & No. \\
\hline Primary treatment & 11 \\
\hline Surgery alone & 14 \\
\hline Surgery + EBRT & Median 66 Gy \\
\hline Radiation dose & 14 \\
\hline Time to relapse & 11 \\
\hline Gender & $57(34-76)$ \\
\hline Male & 15 months (3-40) \\
\hline Female & 15 \\
\hline Median age (range) & 18 \\
\hline Implant location & 7 \\
\hline Oral cavity & \\
\hline Oropharynx & \\
\hline Treatment modality & \\
\hline Radical brachytherapy & MBRT with BRT
\end{tabular}

EBRT - external beam radiation therapy; BRT - brachytherapy by brachytherapy boost. Thus, the study consisted of 18 patients who underwent radical BRT and 7 patients who underwent brachytherapy boost following EBRT. The treatment characteristics are shown in Table 2.

\section{Brachytherapy}

The implant procedure was performed under general anesthesia. A nasogastric tube was placed for feeding during treatment. A straight stainless-steel needle was introduced through the sub-mental skin with respect to the site and traversed through the floor of mouth or implanting organ, exited at the other end of operative bed. Subsequent needles passed next to the first one as needed with respect to number of lines and planes, in order to keep interval distance of $14-16 \mathrm{~mm}$ between them, according to the need to cover the target. A plastic catheter was threaded through each needle and then, the needle was removed, leaving the catheter in place. The number of catheters varied according to the dimension of the target (Figure 1). The plastic catheters were placed in the operative bed as near parallel as possible at 14 to $16 \mathrm{~mm}$ intervals, taking care of peripheral fall-off with a security margin of $10 \mathrm{~mm}$ in all directions around the target, using modified technique. The catheters were held to the skin exit points with plastic buttons $[13,14]$. This implantation technique was used for the various HNC sites.

Prophylactic tracheostomy was not done routinely, except for one patient, where lingual surface of epiglottis was involved. After implantation, all patients underwent a computed tomography (CT) scan, with a slice thickness of $3 \mathrm{~mm}$ for three-dimensional (3D) treatment planning. Intravenous contrast was used when necessary to visualize the carotid vessels. The CT study was transferred to the Flexiplan system (Nucletron, an Elekta company, Elekta AB, Stockholm, Sweden). PTV (planning target volume) and OAR (organ at risk) were contoured and catheters were reconstructed. The treatment planning process was done by computer-assisted dose optimization (Figure 1). The prescribed dose was in the range of 3.5-4.5 Gy per fraction, depending on the site and status

Table 2. Treatment characteristics

\begin{tabular}{lc}
\hline Parameters & Total patient $n=25$ \\
\hline Median number of fractions & 10 (6-12) \\
\hline $\begin{array}{lc}\text { Brachytherapy radiation dose } \\
\text { Radical (median) }\end{array}$ & 40.5 Gy \\
\hline With EBRT & 27 Gy \\
\hline Implant volume (median) & 25 months \\
\hline Median follow-up & 3 \\
\hline Grade of differentiation & 9 \\
\hline Well & 13
\end{tabular}

EBRT - external beam radiation therapy 
of the disease. Fractions were given twice a day with 6 hours apart (median: 10 fractions). The dose parameters were assessed through DVH (dose volume histogram) in percentage. Prescribed and reported doses were specified by $\mathrm{D}_{90}$ (dose received by $90 \%$ of the volume), as determined by DVH. The implant was planned after 2-3 weeks of completion of EBRT. In cases where BRT was used as a boost, median gap between external and implant was 21 days.

The implant tubes were removed after planned BRT doses were delivered. Total dose (EBRT/BRT) was kept within tolerance levels and has been assessed by estimating biologically equivalent doses (BED) $[8,9]$. The median dose with radical BRT was $40.5 \mathrm{~Gy}$, and the dose with BRT boost was $27 \mathrm{~Gy}$. The median dose to primary target volume with EBRT was 50 Gy (range: 46-50 Gy). The median implant volume was $85 \mathrm{cc}$ (range: $38-240 \mathrm{cc}$ ). Overall, the median number of fractions were 10 (range: 6-12).

\section{Follow-up}

Patients underwent follow-up evaluation at every 4 weeks for the first 6 months, every 3 months for the next 6 months, every 6 months for the next 3 years, and annually thereafter. Biopsy was avoided unless it was essential to confirm residual/re-recurrent disease. Overall, follow-up ranged from 8 to 50 months (median: 25 months) for all patients. Twenty patients reached the two-year follow-up and of these, 6 patients reached the five-year follow-up; these patients were alive at the time of reporting in December 2017. The patients were followed-up with routine investigations including complete blood counts, chest X-ray, and ultrasonography of the neck. In suspicious cases, CT of the neck was done.

\section{Statistical analysis}

Statistical analysis was performed using SPSS 17.0 (Statistical Package for Social Sciences 17.0 for Windows) statistical software. Survival results were calculated using the Kaplan-Meier method and log-rank test. The time origin was the date of the first HDR-BRT procedure. The endpoint of overall survival (OS) was death from any cause. The endpoint of disease-free survival (DFS) was any type of recurrence (e.g., failure at the primary site or regional lymph nodes, distant metastasis). The endpoint of interest from local control (LC) was defined as tumor regrowth in the treated area with BRT or in an adjacent region (e.g., failure at the primary site or regional lymph nodes). The patient DFS and OS were calculated from the last date of their follow-up. Toxicity assessment was done using the RTOG toxicity assessment scale, and late toxicities were defined as features persisting or occurring beyond 90 days.

\section{Results}

\section{Overall survival}

The median survival calculated for patients who underwent reirradiation with HDR-BRT at 1- and 2-year overall survival was found to be $77 \%$ and $68 \%$, respectively. The median follow-up was 25 months. There was

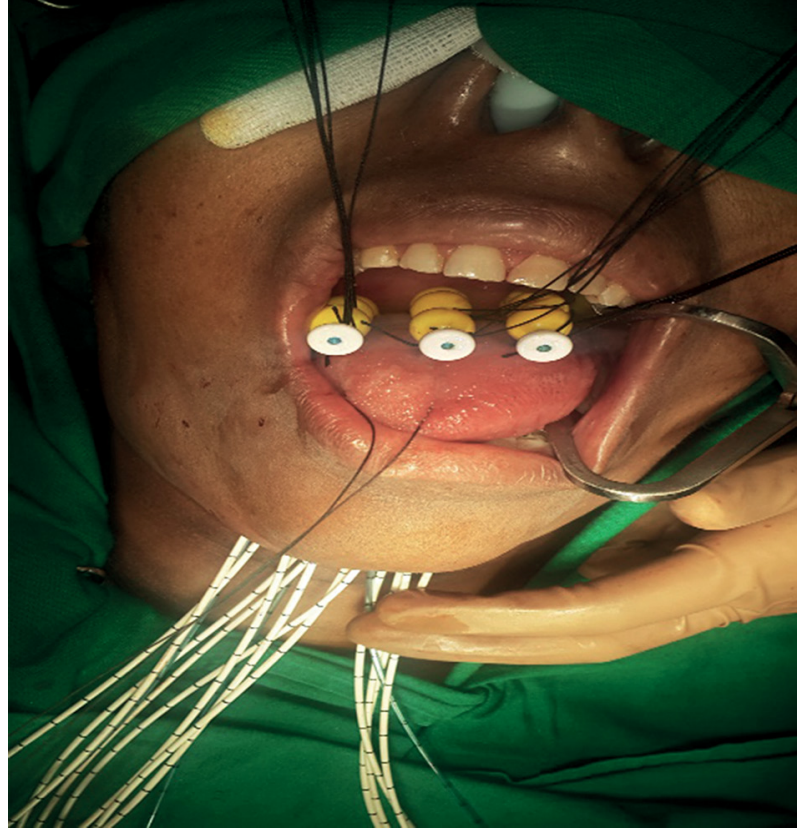

Fig. 1. Plastic bead placement using Bhalavat's technique for interstitial brachytherapy

a difference in the survival rates in radical versus boost arm as shown in Figure 2.

\section{Local control}

The local control rates calculated at 1- and 2-year follow-up were found to be $84 \%$ and $75 \%$, respectively. The median time to development of recurrence was found to be 9 months, and was found to be seen more with a larger tumor implant (CTV $>85 \mathrm{cc}$ ) and more common in the EBRT + BRT arm. The local control rate with radical BRT vs. BRT as boost following EBRT was found to be significant (2-year LCR $62 \%$ vs. $85 \%$; $p<0.02$ ). Similarly, the disease specific survival was found to be $74 \%$ and $67 \%$, respectively, at 1- and 2-year follow-up.

\section{Toxicity}

The late toxicities were assessed as per the Radiation Therapy Oncology Group (RTOG) scale on each follow-up of the patient, and late toxicities were defined

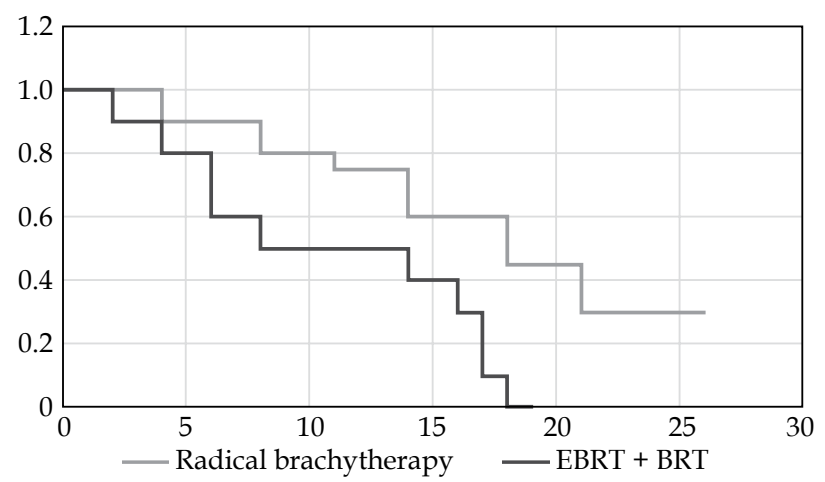

Fig. 2. Survival analysis between radical brachytherapy versus brachytherapy as a boost 
Table 3. Assessment of late toxicities

\begin{tabular}{|c|c|c|c|c|}
\hline Toxicity parameter & Grade 1 & Grade 2 & Grade 3 & Grade 4 \\
\hline Xerostomia & $12 \%$ & $4 \%$ & 0 & 0 \\
\hline Taste alteration & $25 \%$ & $12 \%$ & $2 \%$ & 0 \\
\hline Dysphagia & $8 \%$ & $3 \%$ & 0 & 0 \\
\hline Delayed wound healing & 0 & 0 & 0 & 0 \\
\hline Persistent hoarseness & $3 \%$ & 0 & 0 & 0 \\
\hline Fibrosis & $5 \%$ & $3 \%$ & 0 & 0 \\
\hline
\end{tabular}

as those occurring beyond 90 days. Most of the toxicities noted were grade 1 or 2 and only $2 \%$ grade 3 toxicity were noted in the form of taste alteration. No grade 4 toxicities were noted among the patients. Taste alteration (grade 1 and $2,25 \%$ and $12 \%$ ) and xerostomia (grade 1 and 2,12\% and 4\%) were the common toxicities noted. Other significant toxicities noted were dysphagia (grade 1 and $2,8 \%$ and $3 \%$ ), persistent hoarseness (grade 1 and 2,3\% and 0\%), and fibrosis (grade 1 and 2, $5 \%$ and $3 \%$ ). As compared to other studies, there was no incidence of delayed wound healing, cranial nerve palsy, and osteoradionecrosis associated with brachytherapy procedure noted in our study. The cumulative incidence of grade 1 and 2 toxicities at 2 -years were $12 \%$ and $8 \%$, respectively (Table 3 ).

\section{Prognostic factors}

A univariate Cox analysis on the significant prognostic factors affecting the survival outcomes was performed, including $\mathrm{N}$ stage, dose of first irradiation, interval between the two treatments, and implant volume. Pre-treatment volume of $85 \mathrm{cc}$ was found to be significant for favorable overall survival. The overall survival was found to be 26 months and 12 months $(p=0.02)$, respectively, for implant volume less than and more than $85 \mathrm{cc}$. Time interval since last treatment was found to be significant for the better local control rates. For interval of less than 15 months, the local control rate was for 10 months as opposed to 31 months in patients who had a treatment interval of more than 15 months $(p<0.01)$. As expected, lower nodal status disease had better overall survival outcomes (N0-1 vs. N2-3; $p=0.025$ ). The results are depicted in Figure 3.

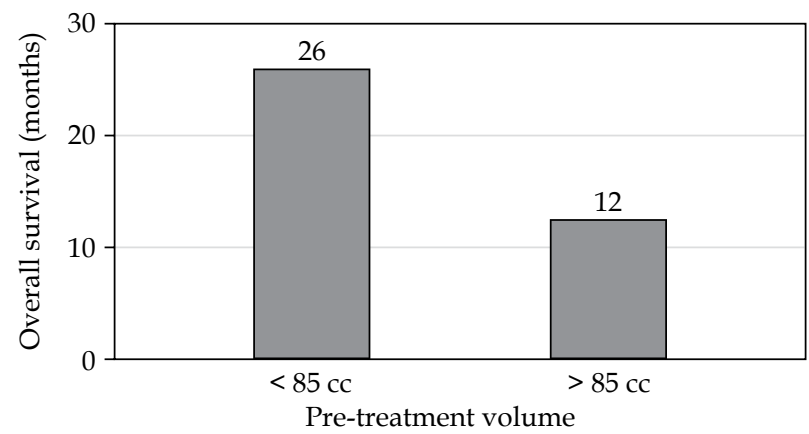

Fig. 3. Prognostic factors on multivariate analysis

\section{Dosimetric outcomes}

On dosimetric assessment, the dose parameters were evaluated, and optimization assessed. Dose heterogeneity was specified by $V_{100}$ (the percentage of implant volume receiving $100 \%$ of the prescribed dose), $V_{150}$ (the percentage of implant volume receiving $150 \%$ of the prescribed dose), and $\mathrm{V}_{200}$ (the percentage of implant volume receiving $200 \%$ of the prescribed dose). In our series, the mean values were: $\mathrm{D}_{90}=4.08 \mathrm{~Gy}$ (range: 3.9-4.5 Gy), equivalent to $90.8 \%$ of the reference dose of $4.5 \mathrm{~Gy}$; $\mathrm{V}_{100}=94.1 \%$ (range: $91-95 \%$ ); $\mathrm{V}_{150}=24.7 \%$ (range: $20-42 \%$ ); $\mathrm{V}_{200}=10.1 \%$ (range: $8-13 \%$ ). The mean values of homogeneity index (HI) and dose non-uniformity ratio (DNR) were estimated to be 0.71 (range: $0.63-0.75$ ) and 0.39 (range: $0.28-0.42$ ), respectively. The various dosimetric outcomes are shown in Tables 4 and 5.

\section{Discussion}

Our retrospective study was aimed to assess the survival rates and toxicity assessment in the patients undergoing reirradiation with HDR-BRT in head and neck malignancies, both as radical treatment and as a boost following EBRT. The literature review has shown that the role of brachytherapy has been used extensively in the past, but non-sorted consensus has not seen brachytherapy as a modality of choice. The literature review with conventional LDR BRT suggests a 2-year LCR of $30 \%$ to $80 \%[15,16,17]$.

On comparison of the results in literature with external beam irradiation, the 2-year local control rate varies from $20 \%$ to $60 \%$ [18,19,20,21]. Even with the advent of technologies and the use of IMRT growing, the toxicity

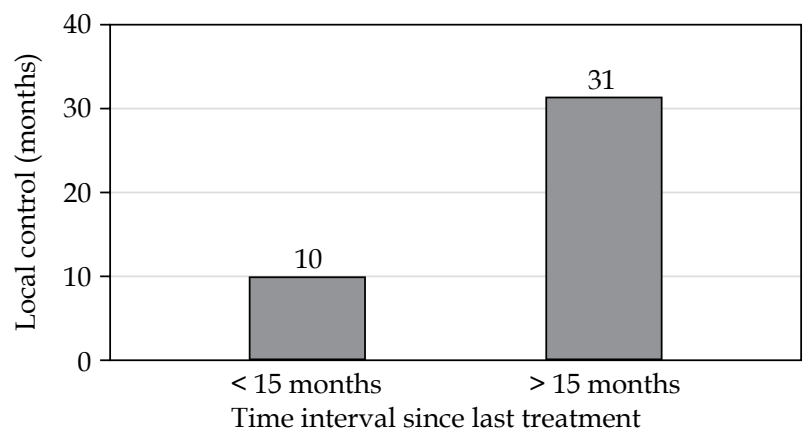


Table 4. Dose delivered and treatment parameters

\begin{tabular}{|c|c|}
\hline Parameters & Values \\
\hline Median implant volume & $85 \mathrm{~cm}^{3}$ \\
\hline EBRT total dose & $\begin{array}{c}\text { mean: } 47.62 \text { Gy } \\
\text { median: } 46 \text { Gy (9-41 Gy) }\end{array}$ \\
\hline BRT dose following EBRT & $\begin{array}{c}\text { mean: } 22.88 \text { Gy } \\
\text { median: } 22.5 \text { Gy (21-65 Gy) }\end{array}$ \\
\hline Radical BRT dose & $\begin{array}{c}\text { mean: } 40.72 \text { Gy } \\
\text { median: } 44.5 \text { Gy (16-53 Gy) }\end{array}$ \\
\hline $\begin{array}{l}\text { Mean interval between EBRT } \\
\text { and BRT }\end{array}$ & 16 days \\
\hline \multicolumn{2}{|l|}{ Post EBRT response } \\
\hline Complete & $81.2 \%$ \\
\hline Partial & $18.8 \%$ \\
\hline Follow-up duration & 25 months (6-84 months) \\
\hline
\end{tabular}

$E B R T$ - external beam radiation therapy; $B R T$ - brachytherapy

levels with brachytherapy have found to be better compared to the newer treatment advents due to the advantage provided by brachytherapy of sharp dose fall beyond the target volume, thereby sparing the normal tissues without compromising on the target volume. The 2 -year overall survival of $68 \%$ and local control rate of $75 \%$ reported in our study has been comparable to the one reported in the literature. Better control rates with radical brachytherapy implants as compared to EBRT + BRT shows that in microscopic residual and small volume disease, interstitial brachytherapy can prove to be an effective salvage option for head and neck malignancies and provide better survival outcomes with effective target coverage. In our study, median survival calculated for patients who underwent re-irradiation with HDR-BRT at 1 and 2-year overall survival was found to be $77 \%$ and $68 \%$, respectively. The median time to development of a recurrence was found to be 9 months, and was found to be more $\mathrm{w}$ frequent in larger tumor implant (CTV $>85 \mathrm{cc}$ ) and more common in the EBRT + BRT arm. The local control rate with radical BRT vs. BRT as boost following EBRT was found to be significant. (2-year LCR $62 \%$ vs. $85 \% ; p<0.02)$. The role of brachytherapy as the sole treatment modality can thus be considered in cases with small tumor volume, which eventually would lead to improved local control, disease specific, and overall survival.

Acute and late toxicities form a major limiting factor for opting for radiation therapy as a salvage treatment option in recurrent head and neck malignancies. The toxicities can outweigh the benefits of therapy and result in negative impact on therapeutic index. In the RTOG 99-11 trial, $8 \%$ grade 5 (fatal) toxicity and $23 \%$ grade 4 acute toxicities were reported [22]. The RTOG 96-10 trial [19] had reported $15 \%$ grade 4 and $7 \%$ grade 5 toxicities. In our series, cumulative incidence of grade 1 and 2 toxicities at 2 -years were $12 \%$ and $8 \%$, respectively, and no grade 4 and 5 toxicities were reported. The use of external beam radiation therapy itself can be a difficult modality to be used for salvage option in recurrent head and neck ma-
Table 5. Dosimetric parameters

\begin{tabular}{lc} 
Dosimetric parameters & \multicolumn{1}{c}{ Value } \\
\hline$D_{90}$ & 4.07 Gy (range: $3.9-4.5$ Gy) \\
\hline$V_{100}$ & $73.33 \%$ (range: $62-95 \%)$ \\
\hline$V_{150}$ & $23.7 \%$ (range: $18-41 \%)$ \\
\hline$V_{200}$ & $12.52 \%$ (range: $11-25 \%)$ \\
\hline Homogeneity index (HI) & 0.71 (range: $0.61-0.75)$ \\
\hline Dose non-uniformity ratio (DNR) & 0.37 (range: $0.29-0.41)$
\end{tabular}

$D_{90}$-dose received by $90 \%$ of the volume; $V x$-volume receiving $x \%$ of the dose

lignancies, as the benefit of therapy can be outweighed by the associated treatment toxicities. Both RTOG studies do not report the delayed toxicities; however, the use of brachytherapy has shown in our series to prevent the severe late toxicities due to the advantage of sharp dose fall off.

The latest report by GEC-ESTRO [23] has reported on the role of HDR-BRT as a salvage option in re-irradiation in the head and neck malignancies. If the patient's ineligible for surgical salvage, brachytherapy is an acceptable option provided that the coverage of the CTV is adequate and there is not advanced bone invasion, fistula, or limited life expectancy. Brachytherapy in previously full course irradiated regions needs to follow the same principles as primary brachytherapy with strict dose and volume constraints [24,25]. Additionally, interstitial brachytherapy can play an important role in the treatment of lymph node recurrences of head and neck cancer. Using image-guided interstitial HDR-BRT for re-irradiation of recurrent lymph node metastases of head and neck cancer, local control probabilities on the order of approximately $60-70 \%$ have been published [26].

The dose of 3.5-4 Gy per fraction has been found to be effective enough to provide good dosimetric coverage and overall survival control, and can be modified as per the stage of the disease with target volume covered. The total dose can be modified as per the treatment interval and the implant volume, which form important prognostic factors for better survival outcomes. Similar dose rates have been used by Hepel et al. [27] and reported a 2-year LCR and survival of $45 \%$ and $37 \%$, respectively. Similar outcomes have also been found in another series with a dose rate of 3.2-4 Gy with favorable survival and toxicity outcomes [28]. In our series, we confirm that the dose of 3.5-4 Gy per fraction to a total dose of 30-40 Gy is effective in providing better survival and late toxicity outcomes. Similar doses have been prescribed in various sites of head and neck malignancies treated with HDR brachytherapy as a boost or as radical intent [29].

\section{Conclusions}

Re-irradiation of recurrent head and neck cancer is a therapeutic challenge. This retrospective study summarizes our experience with HDR brachytherapy treatment of recurrent head and neck cancer. HDR-BRT seems a viable alternative to surgery and radical EBRT. 
Although this was a retrospective series using a small number of patients, our study showed that HDR interstitial brachytherapy demonstrated a better local control probability with an acceptable toxicity in diverse treatment settings. This technique offers dosimetric, radiation safety, and patient comfort advantages.

\section{Disclosure}

Authors report no conflict of interest.

\section{References}

1. Gregoire V, Lefebvre JL, Licitra L et al. ESMO Guidelines Working Group. Squamous cell carcinoma of the head neck: EHNS-ESMO-ESTRO clinical practice guideline for diagnosis, treatment and follow-up. Ann Oncol 2010; 21: 184-186.

2. National Comprehensive Cancer Network. Practice Guidelines: Head and Neck cancer, version 2.2011; 2011 Available from: http://www.nccn.org/professionals/physician_gls/ pdf/head-and-neck.pdf

3. Chopra S, Gupta T, Agarwal JP et al. Re-irradiation in the management of isolated neck recurrences: current status and recommendations. Radiother Oncol 2006; 81: 1-8.

4. Tselis N, Ratka M, Vogt HG et al. Hypofractionated accelerated CT-guided interstitial 192Ir-HDR-Brachytherapy as re-irradiation in inoperable recurrent cervical lymphadenopathy from head and neck cancer. Radiother Oncol 2011; 98: 57-62.

5. McDonald MW, Lawson J, Garg MK et al. ACR appropriateness criteria retreatment of recurrent head and neck cancer after prior definitive radiation expert panel on radiation oncology-head and neck cancer. Int J Radiat Oncol Biol Phys 2011; 80: 1292-1298.

6. Parsons JT, Mendenhall WM, Stringer SP et al. Salvage surgery following radiation failure in squamous cell carcinoma of the supraglottic larynx. Int J Radiat Oncol Biol Phys 1995; 32: 605-609.

7. Teman S, Pape E, Janot F et al. Salvage surgery after failure of very accelerated radiotherapy in advanced head-and-neck squamous cell carcinoma. Int J Radiat Oncol Biol Phys 2005; 62: 1078-1083.

8. Hepel JT, Syed AMN, Puthawala A et al. Salvage high-doserate (HDR) brachytherapy for recurrent head-and-neck cancer. Int J Radiat Oncol Biol Phys 2005; 62: 1444-1450.

9. Narayana A, Cohen GN, Zaider M et al. High-dose rate brachytherapy in recurrent and previously irradiated head and neck cancers-preliminary results. Brachytherapy 2007; 6: 157-163.

10. Safigholi H, Han DY, Mashouf S et al. Direction modulated brachytherapy (DMBT) for treatment of cervical cancer: a planning study with 192Ir, 60Co, and 169Yb HDR sources. Med Phys 2017; 44: 6538-6547.

11. Kollmeier MA, McBrideS, Taggar A et al. Salvage brachytherapy for recurrent prostate cancer after definitive radiation therapy: A comparison of low-dose-rate and high-dose-rate brachytherapy and the importance of prostate-specific antigen doubling time. Brachytherapy 2017; 16: 1091-1098.

12. Han DY, Safigholi H, Soliman A et al. Direction modulated brachytherapy for treatment of cervical cancer. II: Comparative planning study with intracavitary and intracavitary interstitial techniques. Int J Radiat Oncol Biol Phys 2016; 96 : 440-448.

13. Bhalavat RL, Laskar S, Saple MP et al. Technique for interstitial brachytherapy of carcinoma of the tongue. J Brachytherapy Int 1998; 14: 179-183.

14. Pierquin B, Wilson JF, Chassagne D et al. Brachytherapy in base of tongue. In: Modern brachytherapy. Pierquin B, Wil- son JF, Chassagne D (eds.). Masson Publishing Inc., New York 1987; 147.

15. Vikram B, Strong EW, Shah JP et al. Intraoperative radiotherapy in patients with recurrent head and neck cancer. Am J Surg 1985; 150: 485-487.

16. Park RI, Liberman FZ, Lee DJ et al. Iodine- 125 seed implantation as an adjunct to surgery in advanced recurrent squamous cell cancer of the head and neck. Laryngoscope 1991; 101: 405-410.

17. Moscoso JF, Urken ML, Dalton J et al. Simultaneous interstitial radiotherapy with regional or free-flap reconstruction, following salvage surgery of recurrent head and neck carcinoma. Analysis of complications. Arch Otolaryngol Head Neck Surg 1994; 120: 965-972.

18. De Crevoisier R, Bourhis J, Domenge C et al. Full-dose re-irradiation for unresectable head and neck carcinoma: Experience at the Gustave-Roussy Institute in a series of 169 patients. J Clin Oncol 1998; 16: 3556-3562.

19. Spencer SA, Harris J, Wheeler RH et al. RTOG 96-10: re-irradiation with concurrent hydroxyurea and 5-fluorouracil in patients with squamous cell cancer of the head and neck. Int J Radiat Oncol Biol Phys 2001; 51: 1299-1304.

20. Benchalal M, Bachaud JM, Francois P et al. Hyperfractionation in the reirradiation of head and neck cancers. Result of a pilot study. Radiother Oncol 1995; 36: 203-210.

21. Dawson LA, Myers LL, Bradford CR et al. Conformal re-irradiation of recurrent and new primary head-and-neck cancer. Int J Radiat Oncol Biol Phys 2001; 50: 377-385.

22. Horwitz EM, Harris J, Langer CJ et al. Phase II study of paclitaxel and cisplatin in combination with split course concomitant hyperfractionated re-irradiation in patients with recurrent squamous cell cancer of the head and neck: Results of RTOG 99-11. Int J Radiat Oncol Biol Phys 2005; 63: S72-S73.

23. Kovács G, Martinez-Monge R, Budrukkar A et al. GEC-ESTRO ACROP recommendations for head \& neck brachytherapy in squamous cell carcinomas: 1st update - Improvement by cross sectional imaging-based treatment planning and stepping source technology. Radiother Oncol 2017; 122: 248-254.

24. Strnad V, Melzner W, Geiger M et al. Role of interstitial PDR brachytherapy in the treatment of oral and oropharyngeal cancer. A single-institute experience of 236 patients. Strahlenther Onkol 2005; 181: 762-767.

25. Yamazaki H, Yoshida K, Yoshioka Y et al. High dose rate brachytherapy for oral cancer. J Radiat Res 2013; 54: 1-17.

26. Tselis N, Ratka M, Vogt HG et al. Hypofractionated accelerated CT-guided interstitial 192-Ir HDR brachytherapy as re-irradiation in inoperable recurrent cervical lymphadenopathy from head and neck cancer. Radiother Oncol 2011; 98: 57-62.

27. Hepel JT, Syed AM, Puthawala A et al. Salvage high-doserate (HDR) brachytherapy for recurrent head-and-neck cancer. Int J Radiat Oncol Biol Phys 2005; 62: 1444-1450.

28. Martinez-Monge R, Alcalde J, Concejo C et al. Perioperative high-dose-rate brachytherapy in previously irradiated head and neck cancer: Initial results of a phase I/II reirradiation study. Brachytherapy 2006; 5: 203-210.

29. Bhalavat R, Chandra M, Pareek V et al. High-dose-rate interstitial brachytherapy in head and neck cancer: do we need a look back into a forgotten art - a single institute experience. J Contemp Brachytherapy 2017; 9: 124-131. 